\title{
Angewandte Kulturwissenschaften - Überlegungen zum Studienprogramm einer akademischen Disziplin nach dem cultural turn im Hinblick auf berufliche Perspektiven
}

\author{
Applied cultural aerea studies - Considerations \\ of a study agenda in an academic field after the cultural \\ turn with regard to a professional perspective
}

\begin{abstract}
The author tries to attract attention for the careers ant the education of that what we call applied Cultural Studies after the cultural turn in the Humanities. He refers to some empirical studies and tries to put it out how differs the several institutes especially in the German spoken academic world. After all the conclusion of the author could be that there is an big demand for specialists in the area of applied cultural studies. The formation or education has to refer widely to a conception or the terminus of every-day-culture, so that it could be a big help in economic, social and education professions.
\end{abstract}

KEYWORDS: Applied Cultural Studies - cultural turn - academic providers- professional careers humanities.

\section{DER „CULTURAL TURN“ UND DIE FORDERUNG NACH EINER ANGEWANDTEN KULTURWISSENSCHAFT}

Seit mehr als einem halben Jahrhundert lässt sich innerhalb der Geisteswissenschaften von einem "cultural turn" (Bachmann-Medick 2010) analog zum „linguistical turn” zu Anfang des 20. Jahrhunderts (insbesondere durch 
Ludwig Wittgenstein und Ferdinand de Saussure hervorgerufen) sprechen. Zudem versteht sich etwa die Germanistik, insbesondere die germanistische Literaturwissenschaft, zunehmend als Kulturwissenschaft "ohne die philologische Basis des Faches aufzugeben” wie Benthien, Velten (2002: 7) betonen. In jüngeren internationalen Publikationen besteht weitgehend Einigung darüber, dass die "klassischen Philologien“ ohne kulturwissenschaftliche Orientierung nicht mehr vorstellbar sind.

Auch in Arbeitsmarkt bezogenen Zusammenhängen wird dies virulent. So wird etwa in einer Stellenausschreibung der Viadrina in Frankfurt/Oder für zwei Stellen im Bereich romanische und slawische (Fremd-) Literaturwissenschaft vom 08.08.2011 in der Zeit vom Bewerber das Profil einer „kulturwissenschaftlich ausgerichteten interdisziplinär arbeitenden Literaturwissenschaft" gefordert. Diese hier nur exemplarisch genommene Ausschreibung macht auf zwei wichtige, inzwischen schon fast selbstverständliche Tendenzen innerhalb der modernen Philologien aufmerksam: Sie sind ohne starken kulturwissenschaftlichen Bezug nicht mehr vorstellbar, zum anderen sollte dieser Bereich interdisziplinär sein.

Über die Frage, inwieweit es sich hierbei um ein eigenständiges Fach handelt oder inwieweit die Kulturwissenschaft als integraler Bestandteil mutter- und fremdsprachlicher Philologien fungiert, ist an anderer Stelle gehandelt worden (vgl. z.B. Wolting 2012) und soll hier nicht weiter untersucht werden. Festhalten lässt sich aber, dass es nach einer Übersicht im Netz in Deutschland etwa 134 Studienorte (Kulturwissenschaft im engeren Sinne) gibt, die Kulturwissenschaft oft im Zusammenhang mit anderen Fächern wie europäischer Ethnologie o.ä. anbieten, während es in Polen beispielsweise vorzugsweise selbstständige kulturwissenschaftliche Institute gibt (etwa in Poznań oder in Wrocław). Von den erwähnten Möglichkeiten, in Deutschland Kulturwissenschaft zu studieren, bieten nur einige Unis Kulturwissenschaft als "reine Kulturwissenschaft" an wie in Berlin die HU (BA), die Universität Bremen (BA), die Viadrina (BA), die Universität Potsdam (BA, 2. Fach) die Fernuniversität Hagen (BA, unter der Bezeichnung: Kulturwissenschaften), die Universität Leipzig (MA, Kulturwissenschaften), die Universität Regensburg (BA, MA, Vergleichende Kulturwissenschaft (BA, MA), die Universität Saarbrücken (BA, MA, Historisch orientierte Kulturwissenschaft, Diplom) oder die Universität Tübingen (BA, MA, empirische Kulturwissenschaft). ${ }^{1}$

${ }^{1}$ Ansonsten haben deutsche kulturwissenschaftliche Institute jeweils noch eine weitere Bezeichnung wie: Ethik der Textkulturen (Universität Augsburg), Europäische Ethnologie (Universität Bamberg), Kulturwissenschaft mit Schwerpunkt Religion (Universität Bayreuth) und ähnliche, um nur einige wenige zu nennen. 
$\mathrm{Zu}$ fragen wäre nun, ob sich unter Zugrundelegung der Studienprogramme exemplarischer Institute eine Hinwendung zur populären Kultur bzw. Alltagskultur im Sinne des oben erwähnten cultural turn, also eine wirkliche Praxisbezogenheit und Anwendbarkeit der Ausbildung kulturkundlicher Institute, entwickelt hat. Konkretisieren lässt sich die Frage dahingehend, inwieweit sich im Sinne der erwähnten Handlungs- und Anwendungsorientiertheit zugleich immer mehr eine Angewandte Kulturwissenschaft durchsetzt. Wichtigen Aufschluss wäre von einem Bulletin zu erwarten, das mit Unterstützung bundesweiter öffentlicher Forschungsgelder im Hinblick auf die Berufsaussichten von Studierenden der Kulturwissenschaften erstellt wurde, in dem - und das erscheint inzwischen als beinahe selbstverständlich - auf die Notwendigkeit einer Praxisorientierung kulturwissenschaftlicher Ausbildung hingewiesen wird.

\section{BERUFSAUSSICHTEN KULTURWISSENSCHAFTLICHER STUDIERENDER IN DEUTSCHLAND}

Gefördert vom Bundesministerium für Bildung und Forschung gab das Institut für Kulturpolitik der Kulturpolitischen Gesellschaft e.V. in Bonn 2011 ein Bulletin zu einem Projekt "Studium-Arbeitsmarkt Kultur" heraus. Dies war Teil eines breiter angelegten Projekts unter dem Titel „Studium Kultur und der Arbeitsmarkt für kulturvermittelnde und interkulturelle Tätigkeitsfelder“, das vom Juli 2008 bis zum Dezember 2011 durchgeführt wurde.

Insgesamt wurde ein weiter und breiterer Begriff von Kulturvermittlung als oben dargestellt der Untersuchung zugrunde gelegt, so dass im Herbst 2008 von 275 Studiengängen an deutschen Hochschulen (worunter Bereiche wie "angewandte Kulturwissenschaften“, Kultur- und Bildungsmanagement" sowie "Cultural Engineering" zu verstehen sind), nach mehrfachen Aktualisierungen und zum Ende der Studie hin sogar von 350 Studienangeboten der „Kulturvermittlung und Interkultur" in Deutschland ausgegangen wurde (Blumenreich u.a. 2011: 5, siehe dazu die Datenbank „Studium Kultur" auf der Projekthomepage www.studium-kultur.de).

Eine Entwicklung innerhalb der kulturwissenschaftlichen Ausbildung im Hinblick auf den Arbeitsmarkt wurde dabei schon zu Anfang der Darstellung des Projektes erwähnt: „(...) Die Übergänge zwischen den drei Sektoren des Kulturbereiches - öffentlicher Kultursektor, privatwirtschaftlicher Kultursektor und intermediärer Kultursektor sind fließender geworden, die Rahmenbedingungen für die Beschäftigten haben sich verändert" (Blumenreich u.a. 2011: 5). Von daher versucht das Projekt zu hinterfragen, welche 
Kompetenzen im Bereich kulturwissenschaftlicher Berufe heute gefordert sind und inwieweit innerhalb der Curricula der Studienfächer auf diese Bedürfnisse und Fertigkeiten im Hinblick auf den Arbeitsmarkt eingegangen wird.

Konkret bedeutete dies, dass das Projekt die folgenden Ziele verfolgte: „(...) die Erarbeitung eines qualifizierten Überblicks über Studiengänge der Kulturvermittlung, die Ermittlung der Bedürfnisse des Arbeitsmarktes Kultur (Vermittlung) sowie die „Intensivierung des Dialogs zwischen Akteuren des Arbeitsmarktes und der Studienangebote" (vgl. ebd.).

In dem hier maßgeblichen Teil wurden insgesamt 45 Experten aus ganz Deutschland (von Kunstvereinen, Kommunikationszentren, Musikschulen, Theatergemeinden, Literaturbüros, Kunsthallen, Kulturämtern, Kunstmuseen bis zu Arbeitsämtern mit dem Schwerpunkt von Interkultur oder Interkulturelle Kommunikation) befragt. Methodisch wurde dabei noch mal differenziert zwischen Einrichtungen (wie Museum, Theater, Galerie, Volkshochschule, Bibliothek, Archiv/Dokumentationszentrum oder soziokulturelles Zentrum), Tätigkeiten (wie manageriale/geschäftsführende, pädagogische, publizistische/redaktionelle, künstlerische, konzeptionelle/kuratorische, kommunizierende/vermarktende, forschende/wissenschaftliche, informationsverbreitende/dokumentierende, administrative und politische), Bereiche (wie Kunst/Kultur, Bildung, Medien, Wissenschaft, Verwaltung, Politik, Tourismus oder Denkmalpflege), Sektoren (öffentlich, privatwirtschaftlich, intermediär) sowie Sparten (Musik, Bildende Kunst, darstellende Kunst, Literatur, Soziokultur/Kulturelle Bildung, Baukultur, Design, Film und audiovisuelle Medien). Bei der Auswahl der Experten wurde dann noch mal unterschieden im Hinblick auf staatliche (z.B. Musikschule - Musikpädagoge), privatwirtschaftliche (z.B. Musikmanagement oder Konzertagentur) oder zivilgesellschaftliche Einrichtungen (Musikvermittler, Vereingeschäftsführung o.ä).

Als signifikante Kompetenzen wurden von den Befragten (die zum groBen Teil in leitenden Funktionen tätig sind) u.a.: Fachkompetenzen (allgemeine, BWL, pädagogische), Methodenkompetenzen (kulturell-künstlerische Kreativität, organisatorische, Mitarbeiterführung und Multimedia und personale/soziale Kompetenzen (allgemeine, Kommunikations-, Kooperationsund Netzwerkkompetenz herausgestellt (Blumenreich u.a. 2011: 12).

Es dominierten dabei nach Einschätzung der Experten die Methodenkompetenzen (Blumenreich u.a. 2011:16), die dann noch mal unterteilt werden können, je nach Sektor und je nach spezieller Kompetenz: So liegen innerhalb des öffentlichen Kultursektors methodische Kompetenzen (in der Reihenfolge) wie Vermittlung, Informationsbeschaffung und -verarbeitung, kreative Kompetenz, Projektmanagement; Präsentation/Moderation, diszip- 
linübergreifendes Arbeiten, EDV-Programme, Interkulturelle Kompetenz, Fremdsprachen, wissenschaftliches Arbeiten und empirische Sozialforschung weit vorne, im privaten Kultursektor Informationsbeschaffung/Verarbeitung, Projektmanagement; disziplinübergreifendes Arbeiten; Vermittlung: kreative Kompetenz, Präsentation/Moderation, Fremdsprachen, EDV-Programme, interkulturelle Kompetenz, wissenschaftliches Arbeiten und empirische Sozialforschung vorne, während im intermediären Kultursektor ebenfalls Vermittlung, Projektmanagement; Präsentation/Moderation: kreative Kompetenz, Informationsbeschaffung/Verarbeitung; interkulturelle Kompetenz, disziplinübergreifendes Arbeiten, EDV-Programme, Fremdsprachen, wissenschaftliches Arbeiten und empirische Sozialforschung genannt wurden.

Damit lässt sich festhalten, dass die Gewichtigkeit und Bedeutung der verschiedenen Kompetenzen in den unterschiedlichen Kulturbereichen ähnlich gelagert sind und sich nicht signifikant unterscheiden. Aussagekräftiger waren da schon die Aussagen im Hinblick auf den Arbeitsmarkt in zehn Jahren.

Als wichtige Kompetenzen für den Arbeitsmarkt der Zukunft wurden unter anderem genannt: Die Kulturvermittlungskompetenz sowie die Internet/EDV-Kompetenz werden nach Ansichten der Experten in der Zukunft immer mehr an Bedeutung gewinnen, hinzu kommt die kaufmännische Kompetenz, die interkulturelle Kompetenz, das spartenübergreifende Arbeiten sowie die pädagogische Kompetenz (Blumenreich u.a. 2011: 20). Gerade auch der Interkultur (der Verbindung von Kulturvermittlungskompetenz und interkultureller Kompetenz) wird in der Zukunft immer größere Beachtung finden, wie es in der Studie heißt: „Die aktuelle und zukünftige Bedeutung der Interkultur, interkultureller Kompetenzen und speziell ausgebildeter MitarbeiterInnen für Interkultur wird von den ExpertInnen prinzipiell erkannt. Hinsichtlich der praktischen Umsetzung dieser Erkenntnis gibt es aber in vielen Organisationen offensichtlich noch Entwicklungspotenziale" (Blumenreich u.a. 2011: 22).

Damit rückt die aktuelle Ausbildung in den Kulturwissenschaften in den Blickpunkt und nicht zuletzt diejenige der den Studiengang Kulturwissenschaft anbietenden Institute. Als besonders lobenswerte Beispiele werden dabei vor allem die Absolventen des Studiengangs „Kulturwissenschaft und ästhetische Praxis (ehemals „Kulturpädagogik“) der Universität Hildesheim, sowie der Studiengang "Culture, Arts and Media“ (ehemals „angewandte Kulturwissenschaften“) der Universität Lüneburg angeführt.

Als erstes vorläufiges Ergebnis wird dabei festgehalten: „Etwa zwei Drittel der ExperInnen sind mit dem Fach-, methodischen- und personalen/sozialen Kompetenzen ihrer MitarbeiterInnen aus kulturvermittelnden Studiengängen zufrieden oder sogar sehr zufrieden" (Blumenreich u.a. 2011: 25). 
Und in gleichem Maße wird betont: „In eher geringem Maße verweisen die ExpertInnen auf fehlende Fach-, Methoden- und personale/soziale Kompetenzen sowie fehlende praktische Erfahrungen" (ebd., 26). Im übrigen wird dazu darauf verwiesen, dass „mehr als zwei Drittel der befragten Organisationen mit Hochschulen und deren kulturwissenschaftlichen Studiengängen“ (ebd., 29) zusammen arbeiten. Wie sieht nun aber das Verhältnis von Studienangeboten und Arbeitsmarktchancen aus?

Bei der Beantwortung dieser Frage stellte sich heraus, dass viele der Expertinnen und Experten über das Studienangebot allgemein in Deutschland zwar nicht ausreichend informiert, prinzipiell aber der Meinung waren, dass es ein Überangebot im Hinblick auf die zu besetzenden oder freiwerdenden Arbeitsstellen geben würde. Dennoch werden die „Arbeitsmarktchancen der Absolventinnen und Absolventen kulturvermittelnder Berufe insgesamt überwiegend als gut eingeschätzt (in Verbindung etwa mit einem Praktikum, Blumenreich u.a. 2011: 32), nicht zuletzt durch den deutlichen Praxisbezug der einzelnen Studiengänge.

Die Autoren der Studie weisen außerdem darauf hin, dass sie mit der Studie diesen Widerspruch nicht ansatzweise ausreichend begründen können (Blumenreich u.a. 2011: 47) Insgesamt spielt nach Ansicht der Experten eine starke Arbeitsmarktorientierung eine besondere Rolle. „Die besten Methoden zur Erreichung des Ziels der Arbeitsmarktorientierung sehen die ExpertInnen in der Durchführung von gemeinsamen Projekten mit PartnerInnen aus der Praxis und der Einbindung von Lehrkräften aus der Praxis in die Studiengänge“ (ebd., 33) Durch die globale Wirtschaftskrise werden jedoch die Veränderungen auf dem Arbeitsmarkt im Bereich Kulturvermittlung im Hinblick auf die Zukunft als negativer eingeschätzt als noch vor Jahren. Das betrifft vor allem den öffentlichen Sektor (ebd., 36), während die Anzahl der Stellen im privaten und intermedialen Sektor eher sogar gestiegen ist. Zudem wird eine stärkere Durchlässigkeit zwischen den Sektoren beobachtet, wobei vor allem das Thema Interkultur an Bedeutung gewonnen hat (ebd., 37). Ohne Einschränkung wird von einer großen Mehrheit der Befragten (78\%) der Hochschulabschluss als Conditio-sine-qua-non für eine anspruchsvolle Tätigkeit in der Kulturvermittlung betrachtet.

„Die größten beruflichen Zukunftschancen haben nach Meinung der ExpertInnen kulturbezogen ausgebildete ManagerInnen und KulturpädagogInnen. Dahinter stehen vermutlich die von den ExpertInnen in den Interviews häufig thematisierten Überzeugungen, dass es angesichts schrumpfender Ressourcen für die Kultur und einer weiter anhaltenden Diversifizierung der Lebensstile immer stärker darauf ankommt, kulturell-künstlerische Inhalte auf professionelle und effektive Weise zu vermarkten beziehungsweise zu polarisieren, wozu auch gehört, neue Publikumsschichten zu gewinnen" 
(ebd., 45). Die Frage, die sich daraus ergibt und in der Studie nicht abschlieBend beantwortet wird, wäre nun, inwieweit die Institute auf die kommenden Aufgaben der Kulturvermittelnden, die schwerpunktmäßig durch Kenntnisse von Interkultur, ökonomische Kenntnisse (etwa in BWL) etc. umrissen sind, vorbereiten und in dieser Richtung ausbilden können.

Die Quintessenz daraus wäre, dass die wenigen vorhandenen Institute tatsächlich auf die spätere Tätigkeit vorbereiten, eine Meinung, die nicht in allen benachbarten Bereichen so zu beobachten ist, wo etwa auf die Evaluierung interkulturelle Fähigkeiten und Fertigkeiten an deutschen Hochschulen abgezielt wird wie zwei größer angelegte und vom DAAD und vom BMBF (Bundesministerium für Bildung und Forschung) geförderte Studien an der Universität Hildesheim sowie der Universität Köln belegen (das Gesamtprojekt heißt iCommposer", inclusive Communities of Practice of Study, Education and Research), es handelt sich hierbei um zwei Teil(lern-)projekte, die zusammengefasst als Qualiko - Qualifizierung für Interkulturelle Kommunikation bezeichnet werden, wobei sich ersteres auf die Ausbildung von Studierenden bezieht, während das zweite Projekt auf die Hochschulangehörigen zielt (Professoren und Hochschulpersonal, vgl. <http://www.unihildesheim.de/qualiko/qualikolbf.html> sowie <http:/ /www.kopf.ik-bildung. fh-koeln.de/content/e69/e1652/index_ger.html>).

Die Konsequenz aus dem oben Herausgestellten wäre nun, mehr angewandte kulturwissenschaftliche Institute zu gründen, denn auch der Bedarf nach Arbeitsplätzen scheint in den nächsten Jahren größer zu werden. Der Vergleich von Ausbildung und tatsächlichen beruflichen Anforderungen fällt also keineswegs so schlecht aus wie vielleicht zuvor angenommen. Im Folgenden soll noch weiter darauf eingegangen werden, welche Fähigkeiten in der Ausbildung der Zukunft stärker forciert werden sollten. Dabei wollen wir noch genauer die einzelnen Institute für Angewandte Kulturwissenschaft mit einbeziehen. Auch wenn es sich hierbei schwerpunktmäßig um Institute in deutschsprachigen Ländern handelt, so sei dabei doch auch kurz der Blick über die Grenze nach Osten erlaubt.

\section{INSTITUTE ANGEWANDTER KULTURWISSENSCHAFT}

An polnischen Hochschulen gibt es ein Institut der Angewandten Kulturwissenschaft (in Warschau, Instytut Lingwistyki Stosowanej, das im übrigen nur im Englischen diesen Namen trägt, im Polnischen und Deutschen weiterhin als Institut Lingwistyki Stosowanej, als Institut für angewandte Linguistik auf der Homepage aufgeführt wird). Trotz einiger Hochschulen, die den Studiengang in Deutschland als interdisziplinäre Zusatzqualifikati- 
on anbieten (worauf oben schon hingewiesen wurde, gilt als das berühmteste Angebot das ZAK Zentrum für Angewandte Kulturwissenschaft, von dem Mediävisten und Wegbereiter einer Interkulturellen Germanistik Bernd Thum und Germanist und (interkulturelle) Medienwissenschaftler Götz Großklaus gegründet wurde. Beide haben sich ja innerhalb der Gesellschaft einen Namen gemacht und sich durch wegweisende Veröffentlichungen zur Fremdkulturwissenschaft ausgewiesen (vgl. etwa Bernd Thum, Gonthier Louis Fink (Hrsg.) (1993): Praxis interkultureller Germanistik. Forschung - Bildung - Politik. Beiträge zum II. Internationalen Kongress der Gesellschaft für Interkulturelle Germanistik, Straßburg 1991. München: iudicium; Götz Großklaus (1985) Kultursemiotischer Versuch zum Fremdverstehen. In: (Hrsg.) A. Wierlacher, Das Fremde und das Eigene Prolegomena zu einer interkulturellen Germanistik. München: Lang)

Dennoch tut man sich, selbst unter Berücksichtigung der anerkannten, Zeitschrift für Angewandte Kulturwissenschaft Wien (allerdings auch stark am eigentlich überwundenen Hochkulturbegriff orientiert), die in Wien am theaterwissenschaftlichen Institut von Manfred Wagner herausgegeben wird, bislang mit einer Begriffsdefinition nach wie vor schwer. ${ }^{2}$

An deutschen Hochschulen bzw. im deutschsprachigen Raum gibt es wie oben bereits angedeutet einige Institute, die einen Studien- oder Aufbaustudiengang Angewandte Kulturwissenschaft anbieten (z. B. das ZfK der Universität Karlsruhe, die Leuphana-Universität Lüneburg, die Universität Hildesheim, die Universität Münster, die Universität Saarbrücken, die ÖH Klagenfurt oder die TU Dortmund, Angewandte Kultur- und Literaturwissenschaft) oder auch spezielle, anders genannte, aber vom Studienprogramm auf eine ähnliche Ausbildung abzielende Studiengänge wie Kulturmanagement o.ä. wie z.B. an der Franz-Liszt-Musikhochschule in Weimar. Um welche praktischen kulturkundlichen bzw. kulturwissenschaftlichen Inhalte es sich dabei handelt, bleibt allerdings zum Teil äußerst vage und

2 Bis Ende 2011 waren in der seit 2005 im Wiener Präsens-Verlag existierenden Reihe 18 (an der Universität für angewandte Kunst) Ausgaben erschienen, darunter fanden sich Themen wie: Nina Wotapka (2005). Blind Vision - sind Produkte Medien? Der Versuch einer Antwort am Beispiel der Waschmaschine. Band 1; Ruth Mateus-Berr (2007). Fasching und Faschismus. Band 8; Christian Zitt (2008). Vom medialen Körperkult zum gesellschaftlichen Krankheitsbild. Zu den Zusammenhängen zwischen der Darstellung des weiblichen Körpers in Printmedien und Internet und Essstörungen. Band 12; Dagmara Adamska (in Vorbereitung). Polnische Kunst - Zeit der Wende (1980-1990er Jahre). Band 13; Dunja Larise (2009). Mythos Kultur. Eine Kritik des postmodernen Kulturkonzept. Band 15; Sabine Prokop (2010). Bevor Big Brother kam. Über das Fernsehen am Ende des 20. Jahrhunderts. Band 16; Eveline Egger (2010). Kreativität in Kunst und Werbung. Definition und Evaluierung von Hochleistungen. Band 17; Elena Huber (2011). Mode in der Sowjetunion 19171953. Band 18. 
diffus. Der Versuch einer Definition des Begriffs „Angewandte Kulturwissenschaft" wird in der Regel gar nicht oder unzureichend unternommen. Es wird unter anderem von "Kulturarbeit" in einem sehr allgemeinen Sinne gesprochen. Ansonsten wird die Zusammenarbeit mit Kulturinstitutionen im Hinblick auf Projekt-, Kultur- und Innovationsmanagement betont.

\section{KEIN EXPLIZITER BEGRIFF „ANGEWANDTE KULTURWISSENSCHAFT“}

Es ist demnach also davon auszugehen, dass der Begriff Angewandte Kulturwissenschaft nicht ausreichend bzw. gar nicht definitorisch geklärt ist. Deshalb seien hier exkursartig einige Bemerkungen dazu erlaubt. Bis heute kann er (und so wird es in den Studienführern der erwähnten Institute in der Regel auch gemacht), nur über seine Gegenstandsbereiche erklärt werden. Im ganzen Netz (oder in der Sekundärliteratur) findet sich im Gegensatz kein einziger einigermaßen handhabbarer Begriff.

An der Universität Klagenfurt wird zumindest versucht, eine Definition der Studienrichtung zu geben, wenngleich diese sehr im allgemeinen verbleibt: „In den Angewandten Kulturwissenschaften geht es um das Verständnis von menschlichem Handeln, dessen Ergebnissen bzw. um die Schöpfungen des menschlichen Geistes. Kulturwissenschaften sind grundsätzlich interdisziplinär angelegt" (www.studienwahl.at).

Bei der Beschreibung des Studiengangs Angewandte Kulturwissenschaft/Kulturarbeit an der TU Karlsruhe heißt es ebenfalls ziemlich lakonisch: „Gegenstand der Ausbildung im Bereich „angewandte Kulturwissenschaft/Kulturarbeit" sind Theorie und Praxis der Kulturarbeit für staatliche, kommunale und private Träger. Die Ausbildung als Begleitstudium erfolgt auf wissenschaftlicher Grundlage und in Kooperation mit öffentlichen und privaten Kultureinrichtungen."(KIT, Angewandte Kulturwissenschaft (BA.PdF). Die Beschreibung der 14 Einzelbereiche lässt dann aber aufhorchen, wenn man liest, dass innerhalb des Studiums aus Bausteinen wie Grundzüge moderner Kulturinstitutionen, Historische Dimensionen der Kulturpraxis/Kulturelles Erbe, Medienkommunikation, Interkulturelle Kommunikation/Interkulturelles Lernen/Multikulturalität, Wissenschaft und Kultur, Wertewandel/Verantwortungsethik, Kulturpolitik, Kultursoziologie/ /Kulturwissenschaft/Cultural Studies (im engeren Sinne), Kulturökonomik/Kulturmanagement, Architektur und Stadtplanung als Kulturbasis, Arbeitswissenschaft, Theorie und Praxis der Kulturästhetik, Allgemeine Ökologie/Umwelt- und Ressourcenökonomie sowie Technikentwicklung/ /Technikgeschichte ausgewählt werden kann und muss (vgl. ZAK, Studium Generale, Karlsruher Institut für Technologie). 
Diese wenigen Bemerkungen des Begriffs Angewandte Kulturwissenschaft auf der Basis von Studienbeschreibungen sind natürlich nur exemplarisch zu verstehen. Vielleicht wäre von hier aus zu fragen, ob es evtl. andere Bereiche gibt, die den Bereich einer angewandten Kulturwissenschaft abdecken, so wie etwa der erwähnte Bereich Kulturmanagement.

\section{AUSBLICKE UND PERSPEKTIVEN IM HINBLICK AUF BERUFSMÖGLICHKEITEN}

Betrachtet man die Betätigungsfelder der Absolventen der Angewandten Kulturwissenschaft, so fällt auf, dass sie sich größtenteils noch immer in Bereichen im theoretisch zumindest eigentlich überwundenen Begriff der Hochkultur bewegen. Damit fallen sie aber hinter den Anspruch schon einer modernen Kulturwissenschaft nach dem cultural turn zurück. Die Arbeitsgebiete der Absolventen und damit die Studienprogramme einer Angewandten Kulturwissenschaft sollten sich jedoch nicht nur auf die Kulturarbeit, sondern auch auf die Arbeitswelt beziehen und hierin eingebunden sein, wie es ja die Studienprogramme einzelner Institute auch durchaus vorzuweisen haben. Und wie Ernst-Wilhelm Händler für die moderne Literatur gefordert hatte, die Probleme (wie etwa Macht und Geld) der uns am nächsten stehenden Lebenswelt, nämlich der Arbeitswelt in ihre Motivik mit einzubeziehen (vgl. Ernst Wilhelm Händler, "Bitte einen Roman über Banker." Macht und Geld sind elementare menschliche Triebfedern. Warum werden sie in der deutschen Gegenwartsliteratur ausgeblendet? Ein Gespräch mit dem Schriftsteller Ernst-Wilhelm Händler. Die Zeit, Nr. 44. 04.11.2010), so lässt sich dies auch innerhalb der modernen Kulturwissenschaft etwa für das Kulturthema Arbeit fordern (wie es beispielsweise im ZAK in Karlsruhe durchaus zum Studienprogramm gehört):

Auch die Einführung in den Verwaltungsapparat eines Rathauses oder ähnlichem (wie es etwa der Philosoph Richard David Precht für die Schule und im Hinblick auf neue Schulfächer in den Medien gefordert hatte) könnte durchaus eine Aufgabe innerhalb einer angewandten Kulturwissenschaft sein, ebenso die Beurteilung und Einschätzung der Agentur von Arbeit oder von deren Fortbildungsprogrammen durch kommerzielle Agenturen (vgl. z.B. das Werk Schule der Arbeitslosen von Joachim Zelter, Klöpfer, Meyer, Tübingen 2006,), nicht zuletzt in der Mediation (im Sinne von Ausgleich, wie es ja neuerdings heißt) und in der Konfliktforschung, die ja bislang weitgehend eine Domäne der Politologie, Soziologie oder Psychologie ist, sollten es für den Kulturwissenschaftler Arbeitsmöglichkeiten geben. 
Dies würde der Angewandten Kulturwissenschaft nicht nur einen breiteren Qualifikationsrahmen bieten, sondern wäre zum einen auch stärker an einem Alltagskulturbegriff orientiert, hinter den wissenschaftlich im Grunde nicht mehr zurück zu gehen ist, zum anderen an der Arbeitswelt orientiert und somit käme der angewandten Kulturwissenschaft eine bedeutendere Rolle zu.

Insofern wäre dann auch das Definitionsproblem im Hinblick auf diese Art von Angewandter Kulturwissenschaft geklärt: Angewandte Kulturwissenschaft wäre die Heranführung und Information von und über Institutionen alltags- und populärwissenschaftlicher Kultur ( $\mathrm{zu}$ denen im weiten Sinne sicherlich auch Museen etc. gerechnet werden könnte) und die Bereitstellung von Fertigkeiten zum interaktiven Handeln in diesen Bereichen. Damit würde man auch dem Dilemma entraten, nur Zulieferer des Kulturbetriebs zu sein und zugleich würden sich die Möglichkeiten des ausgebildeten Kulturwissenschaftlers auf exorbitante Weise in Richtung und im wahrsten Sinne Arbeitsmarkt vergrößern. Innerhalb dieses Beitrags konnte dabei nur die Richtung der Zukunft dieses Studienfachs angedeutet werden, wichtig wäre jetzt im Anschluss daran, die Arbeitsmöglichkeiten im Konkreten und in Einzelstudien aufzuzeigen. Auf diese Weise wäre im übrigen auch der Forderung nach der „Orientierung Kultur- bzw. Geisteswissenschaften" (vgl. Hartmut Böhme, Peter Matussek, Lothar Müller (Hrsg.) (2002): Orientierung Kulturwissenschaft. Was sie kann, was sie will. Reinbek bei Hamburg: Rowohlt-Taschenbuch-Verlag,; Ludger Heidbrink, Harald Welzer (Hrsg.) (2007): Das Ende der Bescheidenheit. Zur Verbesserung der Geistes- und Kulturwissenschaften. München: C.H. Beck) genüge getan (Benthien und Velten hatten ja drauf hingewiesen, dass Kultur- als erster Teil des Kompositums zum Teil an die Stelle der Geisteswissenschaften getreten ist).

\section{LITERATURVERZEICHNIS}

Bachmann-Medick, D., 2010. Cultural Turns. Neuorientierungen in den Kulturwissenschaften. Reinbek bei Hamburg: Rowohlt.

Benthien, C., Velten, H. R. (Hrsg.), 2002. Germanistik als Kulturwissenschaft. Eine Einführung in neuere Theoriekonzepte. Reinbek bei Hamburg: Rowohlt.

Böhme, H., Matussek, P., Müller, L. (Hrsg.), 2007. Orientierung Kulturwissenschaft. Was sie kann, was sie will. Hamburg.

Gerlach-March, R., 2010. Kulturfinanzierung (Kunst- und Kulturmanagement). Wiesbaden: Verlag für Sozialwissenschaften.

Günter, B., Hausmann, A., 2009. Kulturmarketing (Kunst- ujd Kulturmanagement). Wiesbaden: Verlag für Sozialwissenschaften.

Henrichs, W., Klein, A., 1996. Kulturmanagement von A - Z: 600 Begriffe für Studium und Praxis. München: dtv. 
Höhne, S., 2009. Kunst- und Kulturmanagement. Stuttgart: UTB.

Ickstadt, H. (Hrsg.), 2004. Berufe für Philologen. Darmstadt: Wissenschaftliche Buchgesellschaft (Berufe für Sprach- und Literaturwissenschaftler).

Jameson, F., 1998. The Cultural Turn. Selected Writings on the Postmodern. 1983-1998, London: Verso.

Janson, S., 2004. Studienführer Kulturwissenschaft. Würzburg: Lexika-Verlag.

Kittler, F.A., 2000. Eine Kulturgeschichte der Kulturwissenschaft. München Paderborn: Fink.

Kittsteiner, H.D. (Hrsg.), 2007. Was sind Kulturwissenschaften? 13 Antworten. München Paderborn: Fink.

Klein, A., 2011a. Kompendium Kulturmanagement. Handbuch für Studium und Praxis. München: Vahlen.

Klein, A., 2011b. Das Marketingkonzept für Kulturbetriebe. München: dtv.

Kramer, D., 1997. Von der Notwendigkeit der Kulturwissenschaft. Marburg: Jonas-Verlag.

Mandel, B., 2002. Lust auf Kultur. Karrierewege in das Berufsfeld. Kulturvermittlung. Nürnberg: BW Verlag.

Siebenhaar, K. (Hrsg.), 2003. Karriereziel Kulturmanagement: Studiengänge und Berufsbilder im Profil. Nürnberg: Bildung und Wissen Verlag.

Wolting, S., 2012. „Kein Ort. Nirgends? Überlegungen zur Institutionalisierung einer modernen Kulturwissenschaft an polnischen Hochschulen“. In: Höhne, S. (Hrsg.). Kulturwissenschaft(en). Beiträge verschiedener Disziplinen. Veröffentlichungsband einer Tagung vom 11.-12.11.2011 in Weimar. Weimar (im Druck).

Grundlagenstudien:

Blumenreich, U., Strittmatter, T., Iber-Rebentisch, C., Unter Mitarbeit von Linn Q, (2011). Projekt "Studium-Arbeitsmarkt-Kultur" - Arbeitsmarkt Kulturvermittlung: Ergebnisse der Interviews mit 45 ExpertInnen. Institut für Kulturpolitik der Kulturpolitischen Gesellschaft e.V. Bonn (Webseite: <http://www.studium-kultur.de/>) gefördert durch das Bundesministerium für Bildung und Forschung).

$<$ http://www.uni-hildesheim.de/qualiko/qualikolbf.html> sowie <http://www.kopf.ik-bildung. fh-koeln.de/content/e69/e1652/index_ger.html>. 